\title{
THE FINITE DIMENSIONALITY OF INTEGRAL HOMOLOGY 3-MANIFOLDS
}

\author{
JOHN J. WALSH ${ }^{1}$
}

\begin{abstract}
This note establishes that an integral homology 3-manifold has covering dimension equal to three.
\end{abstract}

The main result is that an integral homology 3-manifold has covering dimension equal to three. Its proof combines a "simple minded" general position argument that uses an elementary property of homology manifolds with the classical result that spaces whose integral cohomological dimension is at most one have covering dimension at most one. Since the class of integral homology 3-manifolds is invariant under $\mathbf{Z}$-acyclic mappings, this proves an alternate proof to that in [KW] that images of cell-like mappings on 3-manifolds are finite dimensional and extends the result to include images of $\mathbf{Z}$-acyclic mappings on 3-manifolds. (Not only does the proof in [KW] differ in that it relies heavily on the "Sphere Theorem" but also there is a serious obstacle to adjusting the proof so that it handles the latter case.)

Since $\mathbf{Z}$-acyclic but not cell-like mappings on 3-manifolds cannot have locally simply connected images [Mc], it is necessary to use Alexander-Spanier cohomology (equivalently, Čech cohomology) and its dual Borel-Moore homology to maintain sufficient generality to capture the result of the second corollary below (see [Bo and $\left.\mathbf{B r}_{1}\right]$ ). A locally compact separable metric space $X$ is an integral homology $n$-manifold provided:

(i) $X$ is cohomologically locally connected over the integers $\left(\mathrm{clc}_{\mathbf{z}}\right)$; i.e., for each $x \in X$ and neighborhood $U$ of $x$, there is a neighborhood $V$ of $x$ with $V \subset U$ such that the inclusion induced homomorphism $i^{*}: \tilde{H}^{*}(U ; \mathbf{Z}) \rightarrow \tilde{H}^{*}(V ; \mathbf{Z})$ is trivial in all dimensions.

(ii) For each $x \in X$,

$$
H_{q}(X, X-\{x\} ; \mathbf{Z}) \simeq \begin{cases}0, & q \neq n, \\ \mathbf{Z}, & q=n .\end{cases}
$$

(iii) The integral cohomological dimension, denoted $\operatorname{dim}_{\mathbf{Z}} X$, is finite; recall that $\operatorname{dim}_{\mathbf{Z}} X$ is the least integer such that $H^{q}(A ; B ; \mathbf{Z})=0$ for all closed pairs $A \supset B$ in $X$ and all $q>\operatorname{dim}_{\mathbf{z}} X$.

Received by the editors July 2, 1982.

1980 Mathematics Subject Classification. Primary 54F45, 55M10; Secondary 07P99.

Key words and phrases. Cohomology 3-manifolds, cohomological dimension, covering dimension, acyclic mapping, cell-like mapping.

${ }^{1}$ Research partially supported by a National Science Foundation Grant.

(C)1983 American Mathematical Society 0002-9939/82/0000-1004/\$01.50 
An assumption of local orientability, traditionally made, was shown in $\left[\mathbf{B r}_{2}\right]$ to be a consequence of these three properties.

The dimension of a space $X$ refers to its covering dimension and is denoted $\operatorname{dim} X$ (see [HW]). It is known that $\operatorname{dim} X=\operatorname{dim}_{\mathbf{z}} X$ when $\operatorname{dim}_{\mathbf{z}} X \leqslant 1$ and when $\operatorname{dim} X<$ $\infty$; but, when $\operatorname{dim} X=\infty$, the equivalence of these dimension theories has not been established (see [Wa] for a further discussion).

MAIN THEOREM. If $X$ is an integral homology 3-manifold, then (the covering dimension) $\operatorname{dim} X=3$.

Before stating two corollaries, we recall that a compactum $A$ is cell-like provided every map of $A$ to every polyhedron is null-homotopic (i.e., $A$ has the shape of a point). A compactum $A$ is $\mathbf{Z}$-acyclic provided it is connected and $H^{q}(A ; \mathbf{Z})=0$ for $q \geqslant 1$. A proper mapping $f: X \rightarrow Y$ for which each point inverse $f^{-1}(y)$ is cell-like (respectively, $\mathbf{Z}$-acyclic) is called a cell-like (respectively $\mathbf{Z}$-acyclic) mapping.

COROLLARY [KW]. The image of a cell-like mapping defined on a 3-manifold is a finite dimensional absolute neighborhood retract.

In the preceding situation, the image is an integral homology 3-manifold and, consequently, is finite dimensional; since the image is also locally homotopically connected, it is an absolute neighborhood retract (see [La, \$3]). In contrast, the image of a $\mathbf{Z}$-acyclic map defined on a 3-manifold is generally not an absolute neighborhood retract but it is an integral homology 3-manifold. (This is essentially Wilder's Monotone Mapping Theorem in [Wi]; verifying that properties (i)-(iii) are preserved involves repeated applications of the Vietoris-Begle Mapping Theorem and occasional uses of duality).

COROLlary. The image of $\mathbf{Z}$-acyclic mapping defined on a 3-manifold is finite dimensional.

Proof of the MaIn TheOrem. Since $X$ is locally compact and locally connected, maps $\alpha: I \rightarrow X$ from the unit interval to $X$ can be approximated arbitrarily closely by maps $\tilde{\alpha}: I \rightarrow X$ such that $\operatorname{dim} \tilde{\alpha}(I) \leqslant 1$. (In fact, $\tilde{\alpha}$ can be chosen to be an embedding but we need not be so discerning.) Specify a countable set of maps $\alpha_{i}: I \rightarrow X$ that comprise a dense subset of the space of maps $\alpha: I \rightarrow X$ endowed with the "supremum metric" topology; further, insist that $\operatorname{dim} \alpha_{i}(I)=1$ for each $i$.

Observe that, for any closed subset $K$ contained in $X \backslash \cup \alpha_{i}(I)$, the set $U \backslash K$ is nonempty and connected whenever $U$ is an open connected subset of $X$. Since the only closed subsets of an integral homology $n$-manifold that have $\operatorname{dim}_{z} \geqslant n-1$ are those that either have nonempty interior or locally separate [Bo, p. 14], it must be that $\operatorname{dim}_{\mathrm{z}} K \leqslant 1$ and, therefore, $\operatorname{dim} K \leqslant 1$ (see [Wa]).

The Sum Theorem [HW, p. 30] assures that $\operatorname{dim} \cup \alpha_{i}(I) \leqslant 1$ and a result of Tumarkin [Na,p. 32] produces a $G_{\delta}$-subset $G$ with $\cup \alpha_{i}(I) \subset G$ and $\operatorname{dim} G \leqslant 1$. Finally, $X \backslash G$ is an $E_{\sigma}$-subset and each closed subset has dimension at most 1; the Sum Theorem assures that $\operatorname{dim}(X \backslash G) \leqslant 1$. Finally, $\operatorname{dim} X \leqslant \operatorname{dim}(X \backslash G)+\operatorname{dim}(G)$ $+1 \leqslant 3\left[\right.$ [HW, p. 28] and, hence, $\operatorname{dim} X=\operatorname{dim}_{\mathbf{z}} X=3$. 


\section{REFERENCES}

[Bo] A. Borel, Seminar On transformation groups, Ann. of Math. Studies, No. 46, Princeton Univ. Press, Princeton, N. J., 1960.

$\left[\mathrm{Br}_{1}\right]$ G. E. Bredon, Sheaf theory, McGraw-Hill, New York, 1967.

$\left[\mathrm{Br}_{2}\right]$, Wilder manifolds are locally orientable, Proc. Nat. Acad. Sci. U.S.A. 63 (1969), 1079-1081.

[HW] W. Hurewicz and H. Wallman, Dimension theory, Princeton Univ. Press, Princeton, N. J., 1941.

[KW] G. Kozlowski and J. Walsh, Cell-like mappings on 3-manifolds, Topology (to appear).

[La] R. C. Lacher, Cell-like mappings and their generalizations, Bull Amer. Math. Soc. 83 (1977), 495-552.

[Mc] D. R. McMillan, Acyclicity in three-manifolds, Bull. Amer. Math. Soc. 76 (1970), 942-964.

[Na] J. Nagata, Modern dimension theory, Interscience, New York, 1965.

[Wa] J. J. Walsh, Dimension, cohomological dimension, and cell-like mappings, Shape Theory and Geometric Topology (S. Mardełić and J. Segal, eds.), Springer-Verlag, New York, 1981.

[Wi] R. L. Wilder, Monotone mappings on manifolds. II, Michigan Math. J. 5 (1958), 19-23.

Department of Mathematics, University of Tennessee, Knoxville, Tennessee 37996 